\title{
CHARACTER AMENABILITY AND CONTRACTIBILITY OF ABSTRACT SEGAL ALGEBRAS
}

\author{
MAHMOOD ALAGHMANDAN, RASOUL NASR-ISFAHANI ${ }^{\bowtie}$ and MEHDI NEMATI
}

\author{
(Received 17 December 2009)
}

Abstract

Let $\mathcal{B}$ be an abstract Segal algebra with respect to $\mathcal{A}$. For a nonzero character $\phi$ on $\mathcal{A}$, we study $\phi$-amenability, and $\phi$-contractibility of $\mathcal{A}$ and $\mathcal{B}$. We then apply these results to abstract Segal algebras related to locally compact groups.

2000 Mathematics subject classification: primary 46H05; secondary 43A07.

Keywords and phrases: character amenable, abstract Segal algebra.

\section{Introduction}

Let $\mathcal{A}$ be a Banach algebra and $\phi \in \sigma(\mathcal{A})$, consisting of all nonzero characters on $\mathcal{A}$. Kaniuth et al. $[7,8]$ introduced and studied the concept of $\phi$-amenability for Banach algebras as a generalization of left amenability of Lau algebras. In fact, $\mathcal{A}$ is called $\phi$-amenable if there exists a bounded net $\left(a_{\alpha}\right)$ in $\mathcal{A}$ such that

$$
\phi\left(a_{\alpha}\right) \rightarrow 1 \text { and }\left\|a a_{\alpha}-\phi(a) a_{\alpha}\right\| \rightarrow 0
$$

for all $a \in \mathcal{A}$. Any such net is called a bounded approximate $\phi$-mean.

Moreover, the notion of (right) character amenability was introduced and studied by Monfared [9]. Character amenability of $\mathcal{A}$ is equivalent to $\mathcal{A}$ being $\phi$-amenable for all $\phi \in \sigma(\mathcal{A})$ and $\mathcal{A}$ having a bounded right approximate identity.

For $\phi \in \sigma(\mathcal{A})$, the notion of $\phi$-contractibility of $\mathcal{A}$ was recently introduced and studied by $\mathrm{Hu}$ et al. [4]. In fact, $\mathcal{A}$ is called $\phi$-contractible if there exists a (right) $\phi$-diagonal for $\mathcal{A}$; that is, an element $\mathbf{m}$ in the projective tensor product $\mathcal{A} \widehat{\otimes} \mathcal{A}$ such that

$$
\phi(\pi(\mathbf{m}))=1 \quad \text { and } \quad a \cdot \mathbf{m}=\phi(a) \mathbf{m}
$$

for all $a \in \mathcal{A}$, where $\pi$ denotes the product morphism from $\mathcal{A} \widehat{\otimes} \mathcal{A}$ into $\mathcal{A}$ given by $\pi(a \otimes b)=a b$ for all $a, b \in \mathcal{A}$.

Several authors have studied various notions of amenability for abstract Segal algebras; see, for example, Samea [13] and Tewari and Parthasarathy [14]. Here, we characterize character amenability and character contractibility of abstract Segal

(C) 2010 Australian Mathematical Publishing Association Inc. 0004-9727/2010 \$16.00 
algebras. We then give some applications of our results to abstract Segal algebras on a locally compact group $G$.

\section{Character amenability and contractibility of abstract Segal algebras}

Let $\mathcal{A}$ be a Banach algebra with the norm $\|\cdot\|_{\mathcal{A}}$. Then a Banach algebra $\mathcal{B}$ with the norm $\|\cdot\|_{\mathcal{B}}$ is an abstract Segal algebra with respect to $\mathcal{A}$ if:

(1) $\mathcal{B}$ is a dense left ideal in $\mathcal{A}$;

(2) there exists $M>0$ such that $\|b\|_{\mathcal{A}} \leq M\|b\|_{\mathcal{B}}$ for all $b \in \mathcal{B}$;

(3) there exists $C>0$ such that $\|a b\|_{\mathcal{B}} \leq C\|a\|_{\mathcal{A}}\|b\|_{\mathcal{B}}$ for all $a, b \in \mathcal{B}$.

We begin this section with the following result.

THEOREM 2.1. Let $\mathcal{A}$ be a Banach algebra and let $\mathcal{B}$ be an abstract Segal algebra with respect to $\mathcal{A}$. Then the following statements are equivalent.

(a) $\mathcal{B}$ is character amenable.

(b) $\mathcal{A}=\mathcal{B}$, and $\mathcal{A}$ is character amenable.

(c) $\mathcal{A}$ is Banach algebra isomorphic to $\mathcal{B}$ and $\mathcal{A}$ is character amenable.

PROOF. (a) $\Rightarrow$ (b). Suppose that $\mathcal{B}$ is character amenable. Then $\mathcal{B}$ has a bounded right approximate identity $\left(e_{\alpha}\right)$. Since $\mathcal{B}$ is an abstract Segal algebra, there exists $C>0$ such that

$$
\|a b\|_{\mathcal{B}} \leq C\|a\|_{\mathcal{A}}\|b\|_{\mathcal{B}}
$$

for all $a, b \in \mathcal{B}$. So, for each $b \in \mathcal{B}$,

$$
\begin{aligned}
\|a\|_{\mathcal{B}}=\lim _{\alpha}\left\|a e_{\alpha}\right\|_{\mathcal{B}} & \leq C\|a\|_{\mathcal{A}} \liminf _{\alpha}\left\|e_{\alpha}\right\|_{\mathcal{B}} \\
& \leq C\left(\sup _{\alpha}\left\|e_{\alpha}\right\|_{\mathcal{B}}\right)\|a\|_{\mathcal{A}} .
\end{aligned}
$$

On the other hand, there exists $M>0$ such that $\|a\|_{\mathcal{A}} \leq M\|a\|_{\mathcal{B}}$. Thus the norms $\|\cdot\|_{\mathcal{A}}$ and $\|\cdot\|_{\mathcal{B}}$ are equivalent on $\mathcal{B}$. Since $\mathcal{B}$ is dense in $\mathcal{A}$, it follows that $\mathcal{A}=\mathcal{B}$ and $\mathcal{A}$ is character amenable.

(b) $\Rightarrow$ (c). Since $\mathcal{B}$ is an abstract Segal algebra with respect to $\mathcal{A}$, there exists $M>0$ such that

$$
\|a\|_{\mathcal{A}} \leq M\|a\|_{\mathcal{B}}
$$

for all $a \in \mathcal{A}$. Thus $\mathcal{B}$ is Banach algebra isomorphic to $\mathcal{A}$ by the open mapping theorem. The implication (c) $\Rightarrow$ (a) is trivial.

Before we give our next result, let us present an elementary lemma.

LEMMA 2.2. Let $\mathcal{A}$ be a Banach algebra and let $\mathcal{B}$ be an abstract Segal algebra with respect to $\mathcal{A}$. Then $\sigma(\mathcal{B})=\left\{\left.\phi\right|_{\mathcal{B}}: \phi \in \sigma(\mathcal{A})\right\}$.

Proof. Since $\mathcal{B}$ is dense in $\mathcal{A}$, it follows that $\left.\phi\right|_{\mathcal{B}} \neq 0$ and so $\left.\phi\right|_{\mathcal{B}} \in \sigma(\mathcal{B})$. Now suppose that $\psi \in \sigma(\mathcal{B})$. Then there exists $b_{0} \in \mathcal{B}$ such that $\psi\left(b_{0}\right)=1$. Thus for each $b \in \mathcal{B}$,

$$
\psi(b)=\psi\left(b b_{0}\right)
$$


By assumption, there exists $C>0$ such that $\left\|b b_{0}\right\|_{\mathcal{B}} \leq C\|b\|_{\mathcal{A}}\left\|b_{0}\right\|_{\mathcal{B}}$ for all $b \in \mathcal{B}$ and consequently

$$
|\psi(b)|=\left|\psi\left(b b_{0}\right)\right| \leq C\|b\|_{\mathcal{A}}\left\|b_{0}\right\|_{\mathcal{B}} .
$$

This shows that $\psi$ is continuous on $\mathcal{B}$ with respect to the norm $\|\cdot\|_{\mathcal{A}}$. Since $\mathcal{B}$ is dense in $\mathcal{A}$, it follows that $\psi$ has a unique extension $\phi \in \sigma(\mathcal{A})$.

Proposition 2.3. Let $\mathcal{A}$ be a Banach algebra and let $\mathcal{B}$ be an abstract Segal algebra with respect to $\mathcal{A}$ and $\phi \in \sigma(\mathcal{A})$. Then $\mathcal{A}$ is $\phi$-amenable if and only if $\mathcal{B}$ is $\left.\phi\right|_{\mathcal{B}^{-}}$ amenable.

Proof. Suppose that $\mathcal{A}$ is $\phi$-amenable. Then there is a bounded approximate $\phi$-mean $\left(a_{\alpha}\right)$ in $\mathcal{A}$. Fix $b_{0} \in \mathcal{B}$ such that $\phi\left(b_{0}\right)=1$ and set

$$
b_{\alpha}:=a_{\alpha} b_{0}^{2} \in \mathcal{B}
$$

for all $\alpha$. Since $\mathcal{B}$ is an abstract Segal algebra with respect to $\mathcal{A}$, there exist $C>0$ and $M>0$ such that for each $b \in \mathcal{B}$,

$$
\begin{aligned}
\left\|b b_{\alpha}-\phi(b) b_{\alpha}\right\|_{\mathcal{B}} & \leq C\left\|b a_{\alpha}-\phi(b) a_{\alpha}\right\|_{\mathcal{A}}\left\|b_{0}\right\|_{\mathcal{A}}\left\|_{b_{0}}\right\|_{\mathcal{B}} \\
& \leq M C\left\|b a_{\alpha}-\phi(b) a_{\alpha}\right\|_{\mathcal{A}}\left\|b_{0}\right\|_{\mathcal{B}}^{2} \rightarrow 0
\end{aligned}
$$

and

$$
\phi\left(b_{\alpha}\right)=\phi\left(a_{\alpha}\right) \rightarrow 1 .
$$

Since $\left(a_{\alpha}\right)$ is $\|\cdot\|_{\mathcal{A}}$-bounded, it follows that $\left(b_{\alpha}\right)$ is $\|\cdot\|_{\mathcal{B}}$-bounded. Thus $\mathcal{B}$ is $\phi_{\mathcal{B}^{-}}$ amenable.

Conversely, suppose that $\mathcal{B}$ is $\left.\phi\right|_{\mathcal{B}}$-amenable. Then there is a bounded approximate $\left.\phi\right|_{\mathcal{B}}$-mean $\left(b_{\alpha}\right)$ in $\mathcal{B}$. Fix $b_{0} \in \mathcal{B}$ such that $\phi\left(b_{0}\right)=1$ and set

$$
a_{\alpha}:=b_{0} b_{\alpha}
$$

for all $\alpha$. Since $\mathcal{B}$ is an abstract Segal algebra, there exists $M>0$ such that for each $a \in \mathcal{A}$,

$$
\begin{aligned}
\left\|a a_{\alpha}-\phi(a) a_{\alpha}\right\|_{\mathcal{A}} & =\left\|a b_{0} b_{\alpha}-\phi(a) b_{0} b_{\alpha}\right\|_{\mathcal{A}} \\
& \leq\left\|a b_{0} b_{\alpha}-\left.\phi(a) \phi\right|_{\mathcal{B}}\left(b_{0}\right) b_{\alpha}\right\|_{\mathcal{A}}+\left\|\left.\phi(a) \phi\right|_{\mathcal{B}}\left(b_{0}\right) b_{\alpha}-\phi(a) b_{0} b_{\alpha}\right\|_{\mathcal{A}} \\
& \leq M\left(\left\|a b_{0} b_{\alpha}-\left.\phi\right|_{\mathcal{B}}\left(a b_{0}\right) b_{\alpha}\right\|_{\mathcal{B}}+|\phi(a)|\left\|\left.\phi\right|_{\mathcal{B}}\left(b_{0}\right) b_{\alpha}-b_{0} b_{\alpha}\right\|_{\mathcal{B}}\right) \\
& \rightarrow 0
\end{aligned}
$$

and

$$
\phi\left(a_{\alpha}\right)=\left.\phi\right|_{\mathcal{B}}\left(b_{\alpha}\right) \rightarrow 1 \text {. }
$$

Since $\|\cdot\|_{\mathcal{A}} \leq M\|\cdot\|_{\mathcal{B}}$, it follows that $\left(a_{\alpha}\right)$ is a $\|\cdot\|_{\mathcal{A}}$-bounded approximate $\phi$-mean in $\mathcal{A}$, and therefore $\mathcal{A}$ is $\phi$-amenable.

As a consequence of Proposition 2.3 we have the following result.

COROLlary 2.4. Let $\mathcal{B}$ be an abstract Segal algebra with respect to a character amenable Banach algebra $\mathcal{A}$. Then $\mathcal{B}$ is $\phi$-amenable for all $\phi \in \sigma(\mathcal{B})$. Moreover, $\mathcal{B}$ is character amenable if and only if $\mathcal{A}=\mathcal{B}$. 
PROPOSITION 2.5. Let $\mathcal{B}$ be an abstract Segal algebra with respect to a Banach algebra $\mathcal{A}$ and $\phi \in \sigma(\mathcal{A})$. Then $\mathcal{A}$ is $\phi$-contractible if and only if $\mathcal{B}$ is $\left.\phi\right|_{\mathcal{B}}$-contractible.

Proof. Suppose that $\mathcal{A}$ is $\phi$-contractible. Then there is a $\phi$-diagonal $\mathbf{m} \in \mathcal{A} \widehat{\otimes} \mathcal{A}$ for $\mathcal{A}$. Thus, $\phi(\pi(\mathbf{m}))=1$ and $a \pi(\mathbf{m})=\phi(a) \pi(\mathbf{m})$ for all $a \in \mathcal{A}$. Since $\mathcal{B}$ is a dense left ideal in $\mathcal{A}$, there exists $b_{0} \in \mathcal{B}$ such that $\phi\left(b_{0}\right)=1$ and so

$$
b_{1}:=b_{0} \pi(\mathbf{m}) \in \mathcal{B} \text {. }
$$

Now, for each $b \in \mathcal{B}$ we have

$$
b b_{1}=\phi(b) b_{1} \quad \text { and } \quad \phi\left(b_{1}\right)=1 .
$$

It is clear that $b_{1} \otimes b_{1} \in \mathcal{B} \widehat{\otimes} \mathcal{B}$ is a $\left.\phi\right|_{\mathcal{B}}$-diagonal for $\mathcal{B}$.

For the converse, suppose that $\mathbf{m} \in \mathcal{B} \widehat{\otimes} \mathcal{B}$ is a $\left.\phi\right|_{\mathcal{B}}$-diagonal for $\mathcal{B}$. Then $\pi(\mathbf{m})$. $\mathbf{m}=\mathbf{m}$ and $a \pi(\mathbf{m}) \in \mathcal{B}$ for all $a \in \mathcal{A}$. We conclude that $\phi(\pi(\mathbf{m}))=1$ and

$$
a \cdot \mathbf{m}=a \cdot(\pi(\mathbf{m}) \cdot \mathbf{m})=a \pi(\mathbf{m}) \cdot \mathbf{m}=\phi(a) \mathbf{m}
$$

for all $a \in \mathcal{A}$; that is, $\mathbf{m}$ is a $\phi$-diagonal for $\mathcal{A}$.

\section{Applications to group algebras}

Let $G$ be a locally compact group with left Haar measure $\lambda_{G}$ and let $L^{p}(G)$, $1 \leq p \leq \infty$, be the usual Lebesgue space with respect to $\lambda_{G}$ as defined in [2]. The convolution product of two measurable functions $f$ and $g$ at $x \in G$ is defined by

$$
(f * g)(x)=\int_{G} f(y) g\left(y^{-1} x\right) d \lambda_{G}(y),
$$

whenever the integral exists. Then $L^{1}(G)$ endowed with the norm $\|\cdot\|_{1}$ and the convolution product $*$ is a Banach algebra, called the group algebra of $G$. Let $\widehat{G}$ denote the dual group of $G$ consisting of all continuous homomorphisms $\rho$ from $G$ into the circle group $\mathbb{T}$, and define $\phi_{\rho} \in \sigma\left(L^{1}(G)\right)$ to be the character induced by $\rho$ on $L^{1}(G)$; that is,

$$
\phi_{\rho}(h)=\int_{G} \overline{\rho(x)} f(x) d \lambda_{G}(x) \quad\left(f \in L^{1}(G)\right) .
$$

It is known that there is no other character on $L^{1}(G)$; that is,

$$
\sigma\left(L^{1}(G)\right)=\left\{\phi_{\rho}: \rho \in \widehat{G}\right\} ;
$$

see, for example, [6, Theorem 2.7.2] or [2, Theorem 23.7].

Recall that $G$ is called amenable if $L^{1}(G)$ is $\phi_{1}$-amenable; or equivalently, there is a bounded approximate $\phi_{1}$-mean in $L^{1}(G)$.

Before we give our first result in the section, recall from [5] that every Segal algebra is an abstract Segal algebra with respect to $L^{1}(G)$ but not conversely; see also [10, 11]. The linear subspace $S^{1}(G)$ of the convolution group algebra $L^{1}(G)$ is said to be a Segal algebra on $G$ if it satisfies the following conditions.

(1) $S^{1}(G)$ is dense in $L^{1}(G)$. 
(2) $S^{1}(G)$ is a Banach space under some norm $\|\cdot\|_{s}$ and for each $f \in S^{1}(G)$

$$
\|f\|_{1} \leq\|f\|_{s} .
$$

(3) $S^{1}(G)$ is left transition invariant and the map $x \mapsto \delta_{x} * f$ of $G$ into $S^{1}(G)$ is continuous.

(4) $\left\|\delta_{x} * f\right\|_{s}=\|f\|_{s}$ for all $f \in S^{1}(G)$ and $x \in G$.

PROPOSITION 3.1. Let $G$ be an amenable locally compact group. Then any abstract Segal algebra $S(G)$ with respect to $L^{1}(G)$ is $\phi$-amenable for all $\phi \in \sigma(S(G))$. Moreover, $S(G)$ is character amenable if and only if $S(G)=L^{1}(G)$.

Proof. Since $G$ is amenable, it follows that $L^{1}(G)$ is amenable, and so $L^{1}(G)$ is character amenable by [9, Corollary 2.4]. The proof is now complete by Corollary 2.4.

EXAMPLE 3.2. Let $G$ be a compact group endowed with the normalized Haar measure. Then the convolution Banach algebra $L^{\infty}(G)$ is a symmetric abstract Segal algebra with respect to $L^{1}(G)$. Since $G$ is amenable, the convolution Banach algebra $L^{\infty}(G)$ is $\phi$-amenable for all $\phi \in \sigma\left(L^{\infty}(G)\right)$ by Proposition 3.1. We can show that $L^{\infty}(G)$ has a right approximate identity if and only if $G$ is finite. To see this, suppose that $L^{\infty}(G)$ has a right approximate identity. Thus $L^{\infty}(G) * L^{\infty}(G)$ is dense in $L^{\infty}(G)$, but it is well known that

$$
L^{\infty}(G) * L^{\infty}(G) \subseteq L^{\infty}(G) * L^{1}(G) \subseteq C(G),
$$

where $C(G)$ is the set of all continuous functions on $G$. This will yield that $C(G)$ is dense in $L^{\infty}(G)$ with the uniform norm. Thus $C(G)=L^{\infty}(G)$ and consequently $G$ is finite by [3, Lemma 37.3]. The converse is clear.

THEOREM 3.3. Let $G$ be a locally compact group and let $\rho \in \widehat{G}$. Then the following statements are equivalent.

(a) $G$ is compact.

(b) All abstract Segal algebras on $G$ are $\phi_{\rho}$-contractible.

(c) There is an abstract Segal algebra on $G$ which is $\phi_{\rho}$-contractible.

Proof. (a) $\Rightarrow$ (b). Fix $\rho \in \widehat{G}$. Since $G$ is compact, it follows that $\rho \in L^{1}(G)$ and $f * \rho=\phi_{\rho}(f) \rho$ for all $f \in L^{1}(G)$ and

$$
\phi_{\rho}(\rho)=\int_{G} \rho \bar{\rho} d \lambda=\int_{G}|\rho|^{2} d \lambda=1 .
$$

It is clear that $\mathbf{m}=\rho \otimes \rho$ is a $\phi_{\rho}$-diagonal for $L^{1}(G)$. Thus $L^{1}(G)$ is $\phi_{\rho}$-contractible and hence any abstract Segal algebra on $G$ is $\phi_{\rho}$-contractible by Proposition 2.5.

(a) $\Rightarrow$ (c). This implication is trivial. 
(c) $\Rightarrow$ (a). Suppose that there is an abstract Segal algebra with respect to $L^{1}(G)$ which is $\phi_{\rho}$-contractible. Then $L^{1}(G)$ is $\phi_{\rho}$-contractible by Proposition 2.5. It follows that there is a $\phi_{\rho}$-diagonal for $L^{1}(G)$, say $\mathbf{m}$. Thus, $\phi_{\rho}(\pi(\mathbf{m}))=1$ and

$$
f * \pi(\mathbf{m})=\phi_{\rho}(f) \pi(\mathbf{m})
$$

for all $f \in L^{1}(G)$. So, if we put

$$
g:=\bar{\rho} \pi(\mathbf{m}) \in L^{1}(G),
$$

then $\phi_{1}(g)=1$ and $f * g=\phi_{1}(f) g$ for all $f \in L^{1}(G)$; indeed,

$$
\begin{aligned}
\rho f * \pi(\mathbf{m}) & =(\rho f * \pi(\mathbf{m})) * \pi(\mathbf{m}) \\
& =\phi_{\rho}(\rho f * \pi(\mathbf{m})) \pi(\mathbf{m}) \\
& =\phi_{1}(f) \pi(\mathbf{m}) .
\end{aligned}
$$

It follows that $G$ is compact; see, for example, [12, Exercise 1.1.7].

COROLlary 3.4. Let $G$ be a locally compact group and let $S(G)$ be an abstract Segal algebra with respect to $L^{1}(G)$ and $\rho \in \widehat{G}$. Then $G$ is amenable if and only if $S(G)$ is $\phi_{\rho}$-amenable.

Proof. Suppose that $S(G)$ is $\phi_{\rho}$-amenable. Then $L^{1}(G)$ is $\phi_{\rho}$-amenable by Proposition 2.3. Thus $L^{1}(G)$ has a bounded approximate $\phi_{\rho}$-mean, say $\left(f_{\alpha}\right)$. Now, we define $h_{\alpha}:=\bar{\rho} f_{\alpha}$ for all $\alpha$. It follows that

$$
\phi_{1}\left(h_{\alpha}\right)=\phi_{\rho}\left(f_{\alpha}\right) \rightarrow 1
$$

and

$$
f * h_{\alpha}=f * \bar{\rho} f_{\alpha}=\bar{\rho}\left(\rho f * f_{\alpha}\right)
$$

for all $f \in L^{1}(G)$. Consequently,

$$
\begin{aligned}
\left\|f * h_{\alpha}-\phi_{1}(f) h_{\alpha}\right\|_{1} & =\left\|f * \bar{\rho} f_{\alpha}-\phi_{1}(f) \bar{\rho} f_{\alpha}\right\|_{1} \\
& =\left\|\rho f * f_{\alpha}-\phi_{1}(f) f_{\alpha}\right\|_{1} \\
& =\left\|\rho f * f_{\alpha}-\phi_{\rho}(\rho f) f_{\alpha}\right\|_{1} \rightarrow 0 .
\end{aligned}
$$

Therefore, $\left(h_{\alpha}\right)$ is a bounded approximate $\phi_{1}$-mean in $L^{1}(G)$, and so $G$ is amenable. The converse is trivial by Proposition 3.1.

Let $G$ be a locally compact group and let $A(G)$ be the Fourier algebra of $G$ as defined in [1]. Then $\sigma(A(G))$ consists of all point evaluations $\phi_{x}(x \in G)$ defined by $\phi_{x}(f)=f(x)$ for all $f \in A(G)$.

THEOREM 3.5. Let $G$ be a locally compact group and let $S A(G)$ be an abstract Segal algebra with respect to $A(G)$. Then the following statements are equivalent.

(a) $G$ is discrete.

(b) $S A(G)$ is $\phi_{x}$-contractible for all $x \in G$.

(c) $S A(G)$ is $\phi_{x}$-contractible for some $x \in G$. 
Proof. Suppose that $G$ is discrete. Then $\chi_{\{x\}} \in A(G)$ for all $x \in G$, where $\chi_{\{x\}}$ is the characteristic function of $\{x\}$. Choose $f_{0} \in S A(G)$ such that

$$
\left\|f_{0}-\chi_{\{x\}}\right\|_{A(G)}<1 / 2 .
$$

Then $\left|f_{0}(x)-1\right|<1 / 2$, and so $f_{0}(x) \neq 0$. Thus,

$$
\chi_{\{x\}}=\frac{1}{f_{0}(x)} f_{0} \chi_{\{x\}} \in S A(G) .
$$

Moreover, $\phi_{x}\left(\chi_{\{x\}}\right)=\chi_{\{x\}}(x)=1$ and, for each $f \in S A(G)$,

$$
f \chi_{\{x\}}=f(x) \chi_{\{x\}}=\phi_{x}(f) \chi_{\{x\}} .
$$

Hence, it is clear that $\chi_{\{x\}} \otimes \chi_{\{x\}}$ is a $\phi_{x}$-diagonal for $S A(G)$, and consequently, $S A(G)$ is $\phi_{x}$-contractible. That is, (a) implies (b). That (b) implies (c) is trivial.

For (c) $\Rightarrow$ (a), suppose that $S A(G)$ is $\phi_{x}$-contractible. Then there is a $\phi_{x}$-diagonal for $S A(G)$, say $\mathbf{m}$. Thus

$$
\pi(\mathbf{m})(x)=\phi_{x}(\pi(\mathbf{m}))=1
$$

and

$$
f \pi(\mathbf{m})=\phi_{x}(f) \pi(\mathbf{m})=f(x) \pi(\mathbf{m})
$$

for all $f \in S A(G)$. Now let $y \in G$ and choose $g \in A(G)$ such that $g(y)=0$ and $g(x)=1$ and also take $h \in S A(G)$ such that $h(x)=1$. Therefore, $h g \in S A(G)$ satisfies $\phi_{x}(h g)=h(x) g(x)=1$, and so

$$
0=h g \pi(\mathbf{m})(y)=\phi_{x}(h g) \pi(\mathbf{m})(y)=\pi(\mathbf{m})(y) .
$$

It follows that $\chi_{\{x\}}=\pi(\mathbf{m})$. Since $\pi(\mathbf{m})$ is a continuous function on $G$, we conclude that $G$ is discrete.

\section{Acknowledgements}

The authors would like to thank the referee for invaluable comments. They also thank the Center of Excellence for Mathematics at the Isfahan University of Technology for kind support.

\section{References}

[1] P. Eymard, 'L'algèbre de Fourier d'un groupe localement compact', Bull. Soc. Math. France 92 (1964), 181-236.

[2] E. Hewitt and K. Ross, Abstract Harmonic Analysis I, Die Grundlehren der Mathematischen Wissenschaften, 115 (Springer-Verlag, Berlin, 1963).

[3] E. Hewitt and K. Ross, Abstract Harmonic Analysis II, Die Grundlehren der Mathematischen Wissenschaften, 152 (Springer-Verlag, New York, 1970).

[4] Z. Hu, M. S. Monfared and T. Traynor, 'On character amenable Banach algebras', Studia Math. 193 (2009), 53-78. 
[5] B. E. Johnson, 'Weak amenability of group algebras', Bull. London Math. Soc. 23 (1991), 281-284.

[6] E. Kaniuth, A Course in Commutative Banach Algebras (Springer, New York, 2009).

[7] E. Kaniuth, A. T. Lau and J. Pym, 'On $\phi$-amenability of Banach algebras', Math. Proc. Cambridge Philos. Soc. 144 (2008), 85-96.

[8] E. Kaniuth, A. T. Lau and J. Pym, 'On character amenability of Banach algebras', J. Math. Anal. Appl. 344 (2008), 942-955.

[9] M. S. Monfared, 'Character amenability of Banach algebras', Math. Proc. Cambridge Philos. Soc. 144 (2008), 697-706.

[10] H. Reiter, $L^{1}$-algebras and Segal Algebras, Lecture Notes in Mathematics, 231 (Springer, Berlin, 1971).

[11] H. Reiter and J. D. Stegeman, Classical Harmonic Analysis and Locally Compact Groups (Clarendon Press, Oxford, 2000).

[12] V. Runde, Lectures on Amenability (Springer, New York, 2002).

[13] H. Samea, 'Essential amenability of abstract Segal algebras', Bull. Aust. Math. Soc. 79 (2009), 319-325.

[14] U. B. Tewari and K. Parthasarathy, 'A theorem on abstract Segal algebras over some commutative Banach algebras', Bull. Aust. Math. Soc. 25 (1982), 293-301.

MAHMOOD ALAGHMANDAN, Department of Mathematical Sciences, Isfahan University of Technology, Isfahan 84156-83111, Iran

e-mail:m.alaghmandan@math.iut.ac.ir

RASOUL NASR-ISFAHANI, Department of Mathematical Sciences, Isfahan University of Technology, Isfahan 84156-83111, Iran

e-mail: isfahani@cc.iut.ac.ir

MEHDI NEMATI, Department of Mathematical Sciences, Isfahan University of Technology, Isfahan 84156-83111, Iran

e-mail:m.nemati@math.iut.ac.ir 